Draft VERSION APRIL 15, 2015

Preprint typeset using LTEX style emulateapj v. 5/2/11

\title{
FINGERING CONVECTION AND CLOUDLESS MODELS FOR COOL BROWN DWARF ATMOSPHERES
}

\author{
P. Tremblin ${ }^{1,2}$ and D. S. Amundsen ${ }^{1}$ and P. Mourier ${ }^{1,3}$ and I. Baraffe ${ }^{1,4}$ And G. Chabrier $^{1,4}$ and B. Drummond ${ }^{1}$ and D. Homeier $^{4}$ and O. \\ VENOT $^{5}$ \\ Draft version April 15, 2015
}

\begin{abstract}
This work aims to improve the current understanding of the atmospheres of brown dwarfs, especially cold ones with spectral type T and Y, whose modeling is a current challenge. Silicate and iron clouds are believed to disappear at the photosphere at the $\mathrm{L} / \mathrm{T}$ transition, but cloudless models fail to reproduce correctly the spectra of T dwarfs, advocating for the addition of more physics, e.g. other types of clouds or internal energy transport mechanisms.

We use a one-dimensional (1D) radiative/convective equilibrium code ATMO to investigate this issue. This code includes both equilibrium and out-of-equilibrium chemistry and solves consistently the $P T$ structure. Included opacity sources are $\mathrm{H}_{2}-\mathrm{H}_{2}, \mathrm{H}_{2}-\mathrm{He}, \mathrm{H}_{2} \mathrm{O}, \mathrm{CO}, \mathrm{CO}_{2}, \mathrm{CH}_{4}, \mathrm{NH}_{3}, \mathrm{~K}, \mathrm{Na}$, and $\mathrm{TiO}$, VO if they are present in the atmosphere.

We show that the spectra of $\mathrm{Y}$ dwarfs can be accurately reproduced with a cloudless model if vertical mixing and $\mathrm{NH}_{3}$ quenching are taken into account. T dwarf spectra still have some reddening in e.g. $J-H$ compared to cloudless models. This reddening can be reproduced by slightly reducing the temperature gradient in the atmosphere. We propose that this reduction of the stabilizing temperature gradient in these layers, leading to cooler structures, is due to the onset of fingering convection, triggered by the destabilizing impact of condensation of very thin dust.
\end{abstract}

Subject headings: Methods: observational — Methods: numerical — brown dwarfs

\section{INTRODUCTION}

Brown dwarfs with effective temperatures $T_{\text {eff }}$ below $\sim 1000 \mathrm{~K}$ (T and Y spectral types) are of great interest to understand the physics of cool atmospheres and pave the way for future studies of cool exoplanets with the James Space Webb Telescope (JWST) and the European Extremely Large Telescope (E-ELT). Detailed spectra can now be obtained in the near-infrared (NIR) for these objects using e.g. the Hubble Space Telescope (HST), or the Gemini Near-Infrared Spectrograph.

The reddening of $\mathrm{M} / \mathrm{L}$ dwarfs $\left(T_{\text {eff }} \approx 2000 \mathrm{~K}\right)$ is thought to be the result of the condensation of silicate dusts and the subsequent cloud formation (Tsuji et al. 1996, Chabrier et al. 2000; Allard et al. 2001). At the L/T transition ( $T_{\text {eff }} \approx$ $1300 \mathrm{~K}$ ), this reddening disappears with a sharp transition as a function of $T_{\text {eff }}$. Given the high amplitude variability observed at the L/T transition (e.g. Radigan 2014), this effect is interpreted as the clouds breaking up and forming holes at the photosphere (e.g. Marley et al. 2010). Yet, the spectral modeling of $\mathrm{T}$ and $\mathrm{Y}$ dwarfs remains a challenge because the IR colors predicted by the cloudless models below $\sim 1000 \mathrm{~K}$ appear too "blue" compared to observations. This additional reddening has recently been interpreted by Morley et al. (2012, 2014) as the emergence of sulfide and chloride clouds $\left(\mathrm{MnS}, \mathrm{Na}_{2} \mathrm{~S}\right.$, and $\mathrm{KCl}$ ). However, even with this cloudy approach, it appears difficult to obtain a good model of Y-dwarf spectra (see

\footnotetext{
tremblin@astro.ex.ac.uk or pascal.tremblin@cea.fr

Astrophysics Group, University of Exeter, EX4 4QL Exeter, UK

2 Maison de la Simulation, CEA-CNRS-INRIA-UPS-UVSQ, USR 3441, Centre d'étude de Saclay, 91191 Gif-Sur-Yvette, France

3 Master ICFP, Département de Physique, Ecole Normale Supérieure, 24 Rue Lhomond, 75005 Paris, France

${ }^{4}$ Ecole Normale Supérieure de Lyon, CRAL, UMR CNRS 5574, 69364 Lyon Cedex 07, France

5 Instituut voor Sterrenkunde, Katholieke Universiteit Leuven, Celestijnenlaan 200D, 3001 Leuven, Belgium
}

for example WISEPC J1217b in Leggett et al. 2015).

In the present paper, we show under which conditions the modeling of $\mathrm{T} / \mathrm{Y}$-dwarf spectra is possible with a cloudless model. In Sect. 2, we describe our 1D numerical code ATMO and we apply it to T and Y dwarf atmospheres in Sect. 3. The spectral modeling of $\mathrm{Y}$ dwarfs is in good agreement with observations when vertical mixing is taken into account. For $\mathrm{T}$ dwarfs, good agreement is obtained when the temperature gradient in the atmosphere is reduced. We discuss these results in Sect. 4 and we suggest that the enhanced cooling in the deep layers of $\mathrm{T}$ dwarfs arises from the onset of fingering convection, resulting from the condensation of some species in very thin dust.

\section{METHOD AND CODE DESCRIPTION}

We have developed a 1D radiative/convective code ATMO solving for the pressure/temperature $(P T)$ structure of an atmosphere, assuming a vertical energy balance between the internal heat flux and the radiative and convective fluxes, and vertical hydrostatic equilibrium. The convection is taken into account using mixing length theory (MLT) as decribed in Henyey et al. (1965) and Gustafsson et al. (2008) with a mixing length of 1.5 times the local pressure scale height.

The $P T$ structure is solved on a cartesian plane-parallel grid for a given effective temperature $T_{\text {eff }}$ and surface gravity $\log g$. The chemistry takes into account $\sim 150$ species at equilibrium with the condensation of $\sim 30$ liquids/solids assuming elemental abundances from Caffau et al. (2011), and is solved by minimization of Gibbs free energy. The references of the thermodynamic data for the gas-phase species can be found in Venot et al. (2012) except for $\mathrm{H}_{2}$ whose thermodynamic data were fitted from the Saumon-Chabrier equation of state (see Saumon et al. 1995). The data for the condensed species can be found in Robie and Waldbaum (1968) and Chase (1998). In the present models, we neglect rain-out processes and clouds. This approximation is critical to as- 
sess the abundances of some refractory elements such as $\mathrm{Na}$, $\mathrm{K}$, and $\mathrm{S}$ because of the different condensation paths that can happen depending on the thermal history of the atmosphere (e.g. $\mathrm{NaAlSi}_{3} \mathrm{O}_{8} / \mathrm{KAlSi}_{3} \mathrm{O}_{8}$ will not form if $\mathrm{Al}$ and $\mathrm{Si}$ are removed at higher temperatures and $\mathrm{Na}$ and $\mathrm{K}$ will condense in $\mathrm{Na}_{2} \mathrm{~S}$ and $\mathrm{KCl}$ ). However in the spectral window of interest, the main absorbers such as $\mathrm{H}_{2} \mathrm{O}, \mathrm{NH}_{3}, \mathrm{CH}_{4}, \mathrm{CO}$ are not affected by this hypothesis and their abundances can even be computed analytically (see Burrows and Sharp 1999). The code is coupled to the kinetic chemical network of Venot et al. (2012), which includes $\sim 1000$ reversible reactions associated with $\sim 100$ species based on $\mathrm{C}, \mathrm{H}, \mathrm{N}, \mathrm{O}$ elements. Vertical mixing is taken into account with a free parameter $K_{z z}$ representing the vertical eddy diffusion coefficient (see Ackerman and Marley 2001). We perform a full chemical network run on the atmosphere by integrating the chemistry in time (usually up to $10^{10}$ seconds) and we reconverged the PT structure of the atmosphere on the fly until both the chemistry and the PT structure are in a steady state. The $P T$ structure can be solved consistently with the out-of-equilibrium chemistry and can have a significant difference if a main absorber is quenched below the photosphere by the vertical mixing. We tested the kinetic chemical network by reproducing the results of Venot et al. (2012) for HD209458b and HD189733b. Our results are also in good agreement with Hubeny and Burrows (2007), especially considering the differences in elemental abundances and opacity line lists. For our case at $T_{\text {eff }}=1000 \mathrm{~K}, \log \mathrm{g}=4.5$ and $\log K_{\mathrm{zz}}=8.0$, we predict a quenched abundance of $\mathrm{CO}$ at $\sim 2 \times 10^{-4}$ and of $\mathrm{NH}_{3}$ at $\sim 7 \times 10^{-6}$, and the model with $T_{\text {eff }}=900 \mathrm{~K}, \log \mathrm{g}=4.5$ and $\log K_{\mathrm{zz}}=8.0$ in Hubeny and Burrows (2007) indicates a CO abundance of $\sim 1.5 \times 10^{-4}$ (fast2 chemical timescale Yung et al. 1988) and a $\mathrm{NH}_{3}$ abundance of $\sim 5 \times 10^{-6}$

The line-by-line radiative transfer has already been described and tested in Amundsen et al. (2014). The differences in radiative flux and heating rate between our line by line code and the radiative scheme used in the global circulation model of the UK Met-Office, the Unifed Model (Amundsen et al. 2014) are generally of the order of a few percent. We use the same opacities for $\mathrm{H}_{2}-\mathrm{H}_{2}, \mathrm{H}_{2}-\mathrm{He}, \mathrm{NH}_{3}, \mathrm{H}_{2} \mathrm{O}, \mathrm{CO}$, TiO and VO (see Table 1 in Amundsen et al. 2014). Methane $\left(\mathrm{CH}_{4}\right)$ has been updated with the new line list from the Exomol project (Yurchenko and Tennyson 2014) and we included the $\mathrm{CO}_{2}$ opacity using the line list from Tashkun and Perevalov (2011) with the line broadening available in the literature (broadening coefficients from Thibault et al. (1992, 2000); Sharp and Burrows (2007); Padmanabhan et al. (2014)). We have also calculated opacities due to the extremely pressurebroadened sodium and potassium doublets using the line profiles of Allard et al. (2007). We have used the correlated- $k$ method described in Amundsen et al. (2014) to speed up the convergence and combined the $k$-coefficients by using the random overlap method for the mixture (Lacis and Oinas 1991). Then we compute the surface spectrum from the converged PT structure using the line by line radiative transfer. The radiative transfer module also includes Rayleigh scattering by $\mathrm{H}_{2}$ and $\mathrm{He}$, using the accelerated $\Lambda$-iteration technique described in Bendicho and Bueno (1995).

\section{APPLICATION TO Y AND T DWARFS}

\section{1. $Y$ dwarfs and $\mathrm{NH3}$ quenching}

Saumon et al. (2006) have already demonstrated that ammonia is well below its chemical equilibrium abundance in $\mathrm{T}$ dwarfs based on the observed strength of th 9-11 $\mu \mathrm{m}$ absorption feature. It has also later been confirmed in the NIR lines of $\mathrm{NH}_{3}$ (e.g. Saumon et al. 2012), and Cushing et al. (2011) confirmed the signature of vertical mixing in all but one of their Y dwarfs by the very absence of more detectable $\mathrm{NH}_{3}$ absorption. It has been expected that absorption by ammonia in the $Y$ band should be present, but so far the data have not identified such a signature (e.g. Fig. 9 in Cushing et al. 2011). It has been also recently proposed by Leggett et al. (2015) that ammonia is depleted in the atmospheres of $\mathrm{Y}$ dwarfs. Using the Y dwarf WISEPC J1217b (Y0, Leggett et al. 2014) as a template, we show in the left panels of Fig. 1 that indeed a low abundance of ammonia is required to reproduce the shape of the $Y$ band flux around $1 \mu \mathrm{m}$ and the correct flux in the $H$ band. Indeed, as shown in Fig. 2, when vertical mixing is taken into account with a diffusion coefficient, the abundance profile of ammonia is quenched at a relatively deep level (50100 bars), resulting in a depletion of ammonia by approximatively a factor 3 , significantly reducing the absorption in the peaks in the $Y$ band. We took a value of $K_{z z}=10^{8} \mathrm{~cm}^{2} \mathrm{~s}^{-1}$ for the eddy diffusion coefficient, which is within the high values explored by Hubeny and Burrows (2007); however the quenching of the $\mathrm{NH}_{3} / \mathrm{N}_{2}$ chemistry is relatively insensitive to the choice of $K_{z z}$ (see Cushing et al. 2011). When we include the vertical mixing, the condensation of $\mathrm{H}_{2} \mathrm{O}$ and $\mathrm{NH}_{3}$ is ignored for simplicity, this approximation is not an issue because the difference with and without condensation at equilibrium is small at these effective temperatures ( $T_{\text {eff }}$ is shifted by $\sim 10 \mathrm{~K})$. Importantly for the color-magnitude diagrams in the IR, the flux in the $H$ band at $1.6 \mu \mathrm{m}$ is increased and matches well the observed spectrum. As shown in Fig. 1, this increase in the flux is sufficient to produce the reddening required in $J-H$ to match the observations. Applying the same physical treatment to the cooler brown dwarf WISEPA J1541 (Y0.5, Cushing et al.2011), we obtain the same significant improvement between observed and synthetic spectra, as shown in the bottom-left panel of Fig. 1. Since we have included the complete chemical network of Venot et al. (2012) in ATMO, we also predict the quenching of $\mathrm{CO}$ and $\mathrm{CO}_{2}$ (see Fig. 2), which will impact the flux at $4.5 \mu \mathrm{m}$.

\subsection{T dwarfs and reduced temperature gradient}

T dwarfs have bluer colors in $J-H$ and $J-K$ than L dwarfs which indicates that the silicate and iron clouds should fall below the photosphere at the L/T transition. However, their spectra still present a reddening compared to cloudless models and Morley et al. (2012) proposed that another type of clouds such as sulfide clouds might be responsible for this residual reddening. We have calculated spectra for the T dwarfs ROSS 458C (Burgasser et al. 2010) and UGPS 0722-05 (Lucas et al. 2010) whose spectra are shown in right panels of Fig. 11. As suggested by Burgasser et al. (2010), we used a supersolar metallicity $([\mathrm{M} / \mathrm{H}]=0.3)$ for ROSS $458 \mathrm{C}$ based on the measured metallicity of its two companions ROSS $458 \mathrm{~A}$ and B. Our cloudless models overestimate the flux in the $Y$ and $J$ bands, yielding significantly bluer colors in $J-H / J-K$ compared to observations. Since the photosphere in the $Y$ and $J$ bands is deeper than the the photosphere in the $H$ and $K$ bands, reducing the temperature gradient in the atmosphere should decrease the $Y / J$ flux compared to the $H / K$ flux. We have tested this idea by artificially modifying the adiabatic index $\gamma=C_{P} / C_{V}$ of the atmosphere. The pressure/temperature profiles obtained with $\gamma=1.2$ for ROSS 458C and $\gamma=1.27$ for UGPS 0722-05, respectively, are displayed in Fig. 3. and the 

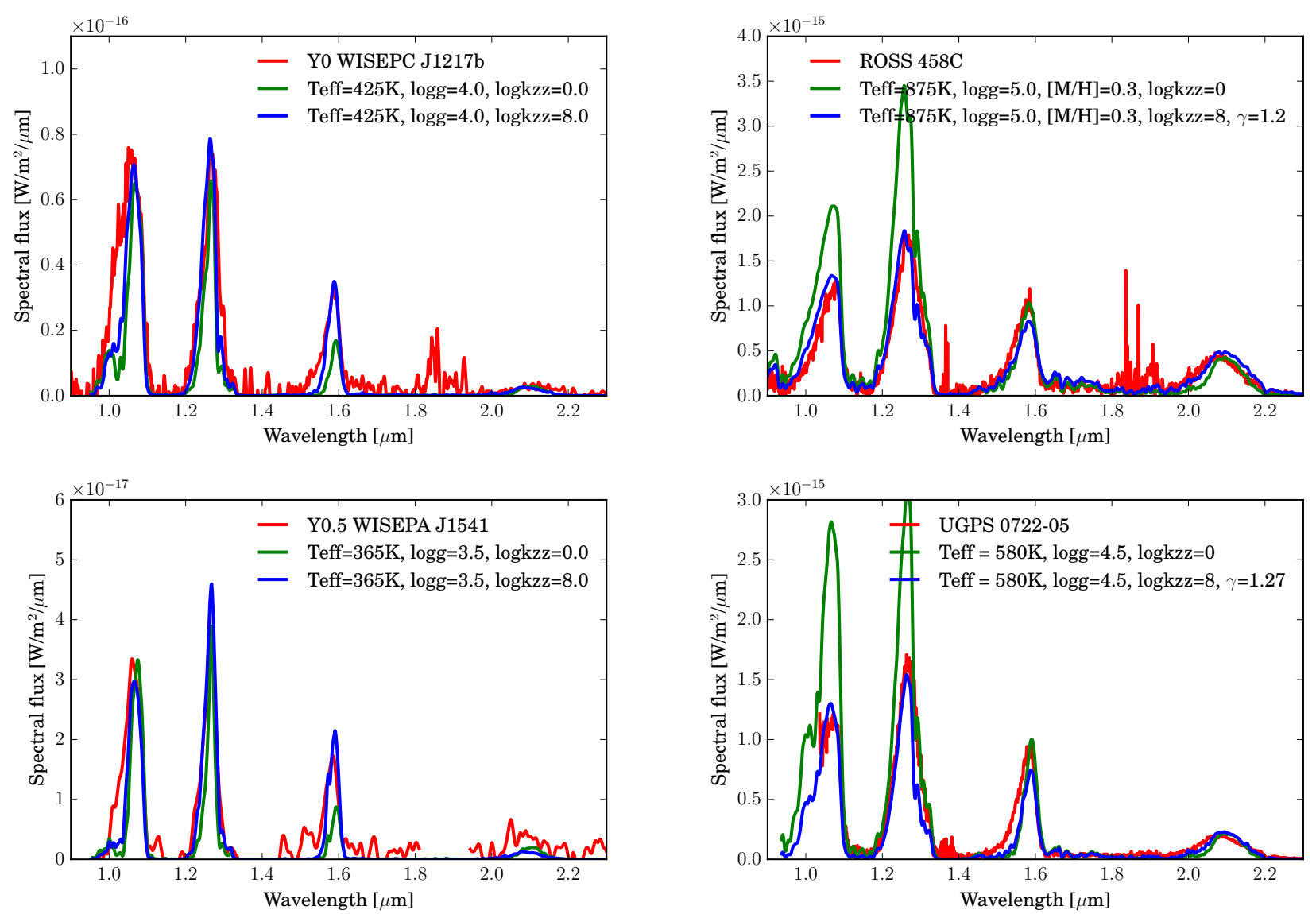

Figure 1. Left panel, top: spectral flux of WISEPC J121756.91+162640.2b (Y0) in red compared to a cloudless model with no vertical mixing (green) and with vertical mixing $\left(K_{z z}=10^{8} \mathrm{~cm}^{2} \mathrm{~s}^{-1}\right.$, blue). Left panel, bottom: similar models for the spectral flux of WISEPA J154151.66225025.2 (Y0.5). The distances are taken from parallax measurements: $10.1_{-1.4}^{+1.9} \mathrm{pc}$ (Dupuy and Kraus 2013) and $5.7_{-0.14}^{+0.15} \mathrm{pc}$ (Tinney et al. 2014), respectively. Right panel, top: spectral flux of ROSS 458C in red compared to a cloudless model (green) and with a reduced temperature gradient (blue). Right panel, bottom: similar models for the spectral flux of UGPS J072227.51-054031.2. The distances are taken from parallax measurements: $11.7_{-0.2}^{+0.2} \mathrm{pc}$ van Leeuwen 2007) and 4.1 $1_{-0.5}^{+0.6} \mathrm{pc}$ (Lucas et al. 2010), respectively. All the models assume a radius of $0.1 \mathrm{R}_{\odot}$, no adjustment of the total flux is used.

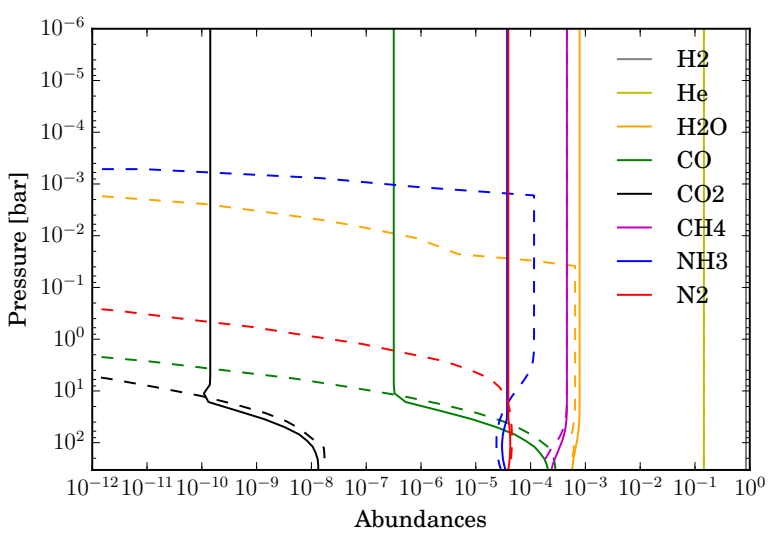

Figure 2. Abundance profiles of the main species in the modeling of WISEPA J1541. The dashed profiles correspond to the model at chemical equilibrium and the solid ones to the model with vertical mixing with $K_{z z}=10^{8} \mathrm{~cm}^{2} \mathrm{~s}^{-1}$.

corresponding spectra in Fig. 1. These models correctly reproduce the spectra and the colors in $J-H / J-K$ without the need to invoke additional cloud opacity. As shown in Fig. 3 the amplitude of the temperature reduction required to match the observed spectra decreases with the effective temperature. Therefore, for $\mathrm{Y}$ dwarfs we found that there is no need to

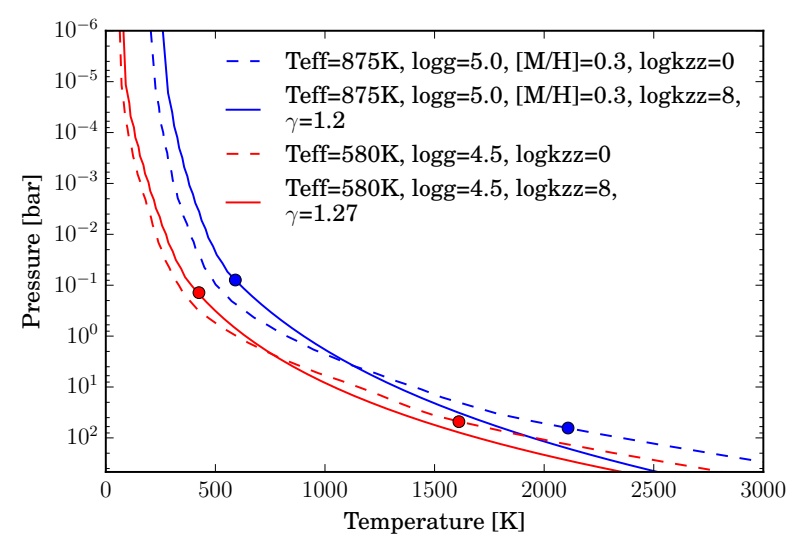

Figure 3. Pressure/temperature profiles of the models of ROSS $458 \mathrm{C}$ and UGPS 0722-05. The solid lines correspond to models with modified adiabatic index $\gamma$. The solid circles indicate the minimum pressures for which convection is present. Without a modified adiabatic index, the convection is overturning, while the modified index, illustrates heat transport by the fingering convection. The change in $K_{\mathrm{zz}}$ has little impact on the PT profile.

adjust the adiabatic index with respect to its expected value $(\sim 1.45)$. The relative importance of the effects of vertical mixing and the reduction of the temperature gradient can be seen on the color-magnitude diagrams in Fig. 4 


\section{DISCUSSION: ATMOSPHERIC FINGERING CONVECTION}

As shown in Sect. 3.1, a coherent cloudless model taking into account vertical mixing in the atmosphere is able to reproduce the near-infrared spectrum of Y dwarfs. Figure 4 shows the color-magnitude diagrams for various cool $\mathrm{T}$ and Y brown dwarfs in $M_{J}$ vs $J-H$ and $M_{Y}$ vs $Y-J$. We have plotted a series of different models at constant log $g=4.5$ as well as the cloudless and sulfide $/ \mathrm{H}_{2} \mathrm{O}$ cloud models of Morley et al. (2014). We display the models with and without vertical mixing and with and without modified adiabatic index to illustrate the relative impacts of these processes. Although the cloudy models do yield redder colors in $J-H$, providing a better match to the observed colors (see Fig. 16 in Morley et al. (2014)), they predict also a large reddening in $Y-J$ which is incompatible with the observations. The problem is even worse at larger gravity $(\log g=5)$. In contrast, our cloudless models reproduce the correct $J-H$ and $Y-J$ colors for Y dwarfs, without any adjustable parameter. Furthermore, as shown in Sect. 3.1, cloudless models consistently taking into account departure from chemical equilibrium due to vertical mixing in the atmosphere nicely reproduce the near-infrared spectrum of Y dwarfs. Our cloudless models are shifted in $J-H$ with respect to the ones of Morley et al. (2014) by nearly one magnitude at $\log g=4.5$ and we would get similar offsets if we compare our models at $\log g=4.5$ to their models at $\log g=5.0$. Part of the shift $(0.3 \mathrm{mag})$ can be explained by changing in our calculations, (i) the elemental abundances to Lodders (2003), (ii) the line list for methane to the STDS line list (Wenger and Champion 1998), (ii) the thermodynamical data of $\mathrm{H}_{2}$ to the one of the JANAF database as used in Morley et al. (2014). The origin of the rest of the shift remains unknown but is probably due to differences in the opacities of the other molecules. It seems more likely, however, that such low-temperature low-mass objects have gravities close to $\log g=4.5$ rather than $\log g=5.0$ (Chabrier and Baraffe 2000).

For T dwarfs, both our cloudless models and the cloudy models reproduce the $J-H$ and $Y-J$ colors. In the former case, as shown in Sect. 3.1, a good match of the spectrum requires a lower value of the atmospheric characteristic adiabatic index (a good match is also obtained in $M_{J}$ versus $J-K)$. This adjustment can thus reflect either the effect of clouds or the signature of an energy transport mechanism that reduces the temperature gradient in the atmosphere. Given the fact that, for $\mathrm{T}$ dwarfs, iron and silicate clouds are expected to have settled below the phostosphere, as mentioned previously, we favor the second suggestion. We suggest that the cooling is due to the onset of fingering (thermohaline) convection in $\mathrm{T}$ dwarf deep atmospheric layers, similar to the one triggered by salt gradients in Earth oceans (e.g. Rahmstorf 2003). In the limit of "infinitely-thin" dust condensation, we have computed the mean-molecular-weight gradient in the atmosphere, $\nabla_{\mu} \sqrt{6}$ assuming that all the condensates remain in the atmosphere in their "mono-molecular" form (e.g., assuming that equilibrium chemistry predicts a given abundance of solid $\mathrm{MgSiO}_{3}$, we compute the mean molecular weight by keeping this abundance with the molecular weight of $\mathrm{MgSiO}_{3}$ ). This mean-molecular-weight gradient should be fairly representative of $\nabla_{\mu}$ in the presence of small grains if the grain formation process does not significantly change throughout the atmosphere. As shown in Rosenblum et al. (2011) (see also Leconte and Chabrier 2012), the value of the dimen-

$$
{ }^{6} \nabla_{X}=\mathrm{d} \ln (X) / \mathrm{d} \ln (P)
$$

sionless parameter $R_{0}=\left(\nabla_{T}-\nabla_{\mathrm{ad}}\right) / \nabla_{\mu}$ determines the extent of the overturning convection zone $\left(R_{0}<1\right)$. In case the mean molecular weight increases with height in a stable atmosphere $\left(\nabla_{\mu}<0\right.$, and $\left.\left(\nabla_{T}-\nabla_{\mathrm{ad}}\right)<0\right)$, as in the present context, the possible existence of fingering convection is determined by the relation $1<R_{0}<1 / \tau$, where $\tau=\kappa_{\mu} / \kappa_{T}$ defines the inverse Lewis number, i.e. the ratio of the molecular diffusivity $\kappa_{\mu}$ to the radiative thermal diffusivity $\kappa_{T}=16 \sigma T^{3} /\left(3 \kappa \rho^{2} C_{p}\right)$. This is the opposite situation to oscillatory double-diffusive convection, due to a positive molecular weight gradient and a destabilizing temperature gradient, suggested to occur in some giant planet interiors (Leconte and Chabrier 2012, 2013). Figure 5 shows the atmospheric profiles of $1 /\left(\tau R_{0}\right)$ for ROSS 458C and WISEPA J1541, taking into account the molecular diffusivity of $\mathrm{MgSiO}_{3}(\mathrm{~s})$ and assuming that the thermal diffusivity is dominated by the radiative processes $\left(\kappa_{T}\right.$ varies from $\sim 10^{-1} \mathrm{~cm}^{2} \mathrm{~s}^{-1}$ at $1 \mathrm{kbar}$ to $\sim 3 \times 10^{5} \mathrm{~cm}^{2} \mathrm{~s}^{-1}$ at $\left.1 \mathrm{bar}\right)$. As seen, fingering convection (i.e. $1 /\left(\tau R_{0}\right)>1$ ) is predicted to occur in some parts of these atmospheres. The magnitude of the mean-molecular-weight gradient is modest: $\left|\nabla_{\mu}\right| \sim 10^{-4}-10^{-5}$ for ROSS $458 \mathrm{C}$. Interestingly, while the extension of the fingering-convection zone is very large for ROSS $458 \mathrm{C}$, it remains relatively small for the Y dwarf WISEPA J1541. This is fully consistent with the fact that we need a lower adiabatic index for $\mathrm{T}$ dwarfs whereas this modification is not necessary for $\mathrm{Y}$ dwarfs.

The transport mechanism needed to lower the temperature gradient has to be efficient to transport energies at the level of the internal flux of the object. The possibility that fingering convection could carry out such energy fluxes remains to be demonstrated. Also the mixing induced by the convective fingers will reduce the destabilizing mean-molecular-weight gradient. A consistent theory of fingering convection in our model is needed in order to assess the possibility to have a self-sustained steady-state energy flux from the convection. Nevertheless our results strongly suggest that fingering convection could occur in $\mathrm{T}$ dwarf atmospheres due to the destabilizing effect of dust condensation in the stably stratified part of the atmosphere, potentially impacting the energy transport in these objects compared to both warmer and cooler objects and leading to efficient cooling of their deep atmospheric layers. It can be expected that the increase of the convective energy transport will reduce the radiative flux in the corresponding region, hence reducing the temperature gradient in the atmosphere, as needed for the reddening in $J-H$.

\section{CONCLUSIONS}

In the present paper, we have shown that

- Cloudless atmosphere models can reproduce the observed infrared Y dwarf spectral energy distribution, provided vertical mixing and out-of-equilibrium chemistry are properly taken into account to correctly predict the quenching of ammonia at deep levels, modifying its abundance profile.

- Cloudless models for T dwarfs with a reduced temperature gradient in the atmosphere correctly reproduce observed fluxes and colors. Such a reduced gradient can be obtained by a modification of the adiabatic index in the atmosphere and could reflect either the effect of clouds or of another type of energy transport in the atmosphere. If clouds are effectively responsible for the reddening, the modification of the adiabatic index is an 

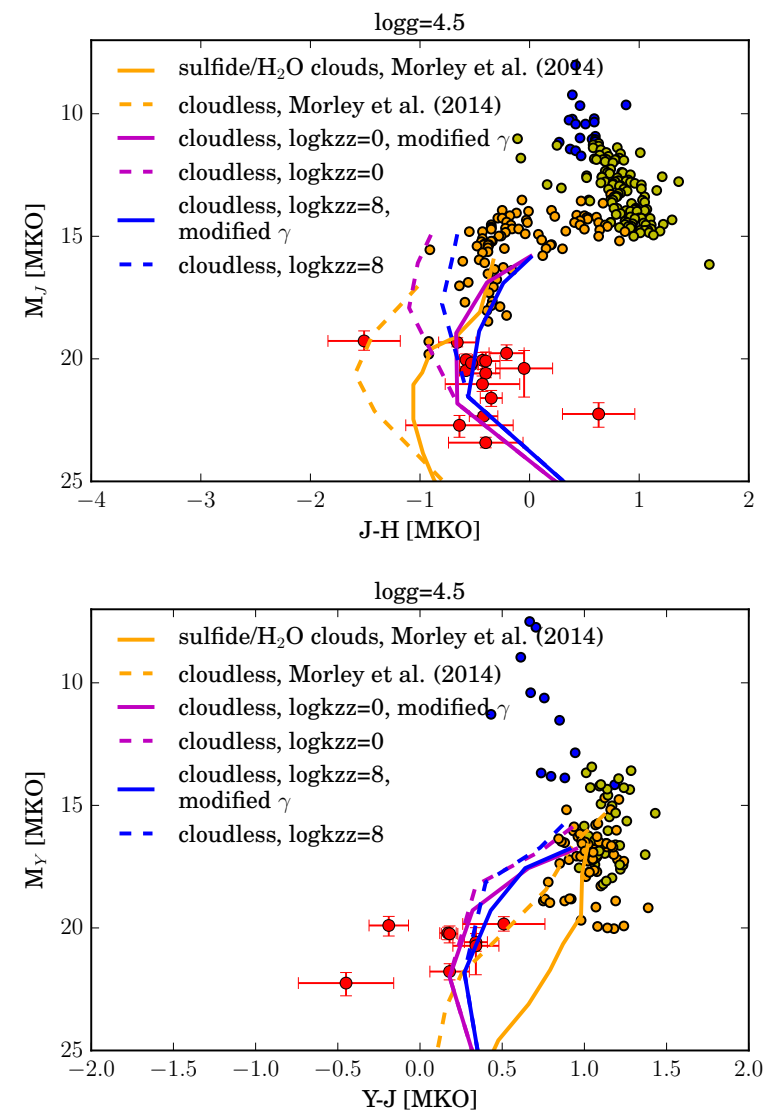

Figure 4. Color magnitude diagrams (top: J-mag vs $J-H$, bottom: Ymag vs $Y-J$ ) for $\mathrm{T} / \mathrm{Y}$ brown dwarfs. The Y-dwarf photometry is from Dupuy and Kraus (2013); Beichman et al. (2014) and the L/T/M from Dupuy and Liu (2012); Faherty et al. (2012). The modified adiabatic index is $\gamma=(1.15,1.2,1.25)$ for $T_{\text {eff }}=(1000 \mathrm{~K}, 800 \mathrm{~K}, 600 \mathrm{~K})$, no modification for $T_{\text {eff }}<600 \mathrm{~K}$

easy way to mimic the effect and could be used to better constrain cloud models that are currently used.

- We suggest that fingering convection could be responsible for such a reduced temperature gradient. We demonstrate that the condensation of very thin dust under typical $\mathrm{T}$ dwarf atmosphere conditions could trigger this mechanism and that the extent of this process decreases with decreasing effective temperature, essentially vanishing for Y dwarf atmosphere conditions.

Fundamental physical mechanisms such as atomic diffusion and hydrodynamical instability might thus take place in cold brown dwarf atmospheres and play a major role in their spectral evolution. Future observations of cool objects with SPHERE, GPI, and JWST combined with comparisons with the different models should enable us to distinguish between these two effects: presence of clouds or reduced temperature gradient in $\mathrm{T}$ dwarf atmospheres. Our ability to constrain which physics is indeed present in cool brown dwarf atmospheres will bear important consequences for the future understanding of cool exoplanet spectra.

We thank Adam Burgasser, Sandy Leggett, Philip Lucas, Mike Cushing, and Chas Beichman for providing their data. The calculations for this paper were performed on the DiRAC Complexity machine, jointly funded by STFC and the Large

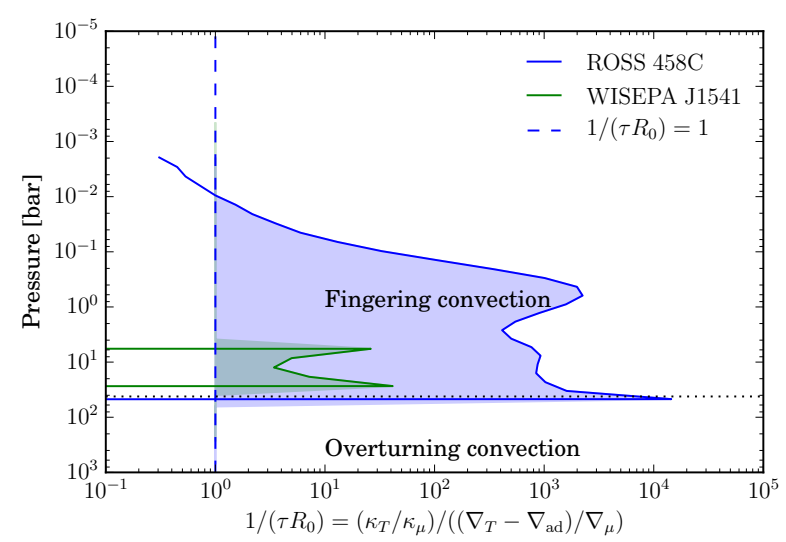

Figure 5. Profile of $1 /\left(\tau R_{0}\right)$ for ROSS $458 \mathrm{C}$ and WISEPA J1541. Fingering (thermohaline) convection is possible where $1 /\left(\tau R_{0}\right)>1$. The dotted horizontal line shows the limit between the overturning and fingering convection zones for ROSS 458C.

Facilities Capital Fund of BIS, and the University of Exeter Supercomputer, a DiRAC Facility jointly funded by STFC, the Large Facilities Capital Fund of BIS, and the University of Exeter. This work is partly supported by the European Research Council under the European Community's Seventh Framework Programme (FP7/20072013 Grant Agreement No. 247060). Part of this work is supported by the Royal Society award WM090065 and the consolidated STFC grant ST/J001627/1. O.V. acknowledges support from the KU Leuven IDO project IDO/10/2013 and from the FWO Postdoctoral Fellowship programme.

\section{REFERENCES}

A. S. Ackerman and M. S. Marley. Precipitating Condensation Clouds in Substellar Atmospheres. ApJ, 556(2):872-884, Aug. 2001.

F. Allard, P. H. Hauschildt, D. R. Alexander, A. Tamanai, and A. Schweitzer The Limiting Effects of Dust in Brown Dwarf Model Atmospheres. ApJ, 556(1):357-372, July 2001.

N. F. Allard, F. Spiegelman, and J. F. Kielkopf. Study of the K-H quasi-molecular line satellite in the potassium resonance line. $A \mathcal{E} A, 465$ (3):1085-1091, Apr. 2007.

D. S. Amundsen, I. Baraffe, P. Tremblin, J. Manners, W. Hayek, N. J. Mayne, and D. M. Acreman. Accuracy tests of radiation schemes used in hot Jupiter global circulation models. AEFA, 564:59, Apr. 2014.

C. Beichman, C. R. Gelino, J. D. Kirkpatrick, M. C. Cushing, S. Dodson-Robinson, M. S. Marley, C. V. Morley, and E. L. Wright. WISE Y Dwarfs as Probes of the Brown Dwarf-Exoplanet Connection. ApJ, 783(2):68, Mar. 2014.

P. F. Bendicho and J. T. Bueno. A novel iterative scheme for the very fast and accurate solution of non-LTE radiative transfer problems. ApJ, 455: 646-657, 1995.

A. J. Burgasser, R. A. Simcoe, J. J. Bochanski, D. Saumon, E. E. Mamajek, M. C. Cushing, M. S. Marley, C. McMurtry, J. L. Pipher, and W. J. Forrest. Clouds in the Coldest Brown Dwarfs: Fire Spectroscopy of Ross 458C. ApJ, 725(2):1405-1420, Dec. 2010.

A. Burrows and C. M. Sharp. Chemical Equilibrium Abundances in Brown Dwarf and Extrasolar Giant Planet Atmospheres. ApJ, 512(2):843-863, Feb. 1999.

E. Caffau, H. G. Ludwig, M. Steffen, B. Freytag, and P. Bonifacio. Solar Chemical Abundances Determined with a CO5BOLD 3D Model Atmosphere. Solar Physics, 268(2):255-269, Feb. 2011.

G. Chabrier and I. Baraffe. Theory of Low-Mass Stars and Substellar Objects. Annual Review of Astronomy and Astrophysics, 38:337-377, 2000.

G. Chabrier, I. Baraffe, F. Allard, and P. Hauschildt. Evolutionary Models for Very Low-Mass Stars and Brown Dwarfs with Dusty Atmospheres. ApJ, 542(1):464-472, Oct. 2000.

M. W. Chase, Jr. NIST-JANAF Thermochemical Tables. New York, 1998. 
M. C. Cushing, J. D. Kirkpatrick, C. R. Gelino, R. L. Griffith, M. F. Skrutskie, A. Mainzer, K. A. Marsh, C. A. Beichman, A. J. Burgasser, L. A. Prato, R. A. Simcoe, M. S. Marley, D. Saumon, R. S. Freedman, P. R. Eisenhardt, and E. L. Wright. The Discovery of Y Dwarfs using Data from the Wide-field Infrared Survey Explorer (WISE). ApJ, 743(1): 50, Dec. 2011.

T. J. Dupuy and A. L. Kraus. Distances, Luminosities, and Temperatures of the Coldest Known Substellar Objects. Science, 341(6):1492-1495, Sept. 2013.

T. J. Dupuy and M. C. Liu. The Hawaii Infrared Parallax Program. I. Ultracool Binaries and the L/T Transition. The Astrophysical Journal Supplement, 201(2):19, Aug. 2012.

J. K. Faherty, A. J. Burgasser, F. M. Walter, N. Van der Bliek, M. M. Shara, K. L. Cruz, A. A. West, F. J. Vrba, and G. Anglada-Escudé. The Brown Dwarf Kinematics Project (BDKP). III. Parallaxes for 70 Ultracool Dwarfs. ApJ, 752(1):56, June 2012.

B. Gustafsson, B. Edvardsson, K. Eriksson, U. G. Jørgensen, Å. Nordlund, and B. Plez. A grid of MARCS model atmospheres for late-type stars. I. Methods and general properties. AEA A, 486(3):951-970, Aug. 2008.

L. Henyey, M. S. Vardya, and P. Bodenheimer. Studies in Stellar Evolution. III. The Calculation of Model Envelopes. ApJ, 142:841, Oct. 1965.

I. Hubeny and A. Burrows. A Systematic Study of Departures from Chemical Equilibrium in the Atmospheres of Substellar Mass Objects. ApJ, 669(2):1248-1261, Nov. 2007.

A. A. Lacis and V. Oinas. A description of the correlated $\mathrm{k}$ distribution method for modeling nongray gaseous absorption, thermal emission, and multiple scattering in vertically ... J. Geophys. Res., 96:9027-9063, 1991.

J. Leconte and G. Chabrier. A new vision of giant planet interiors: Impact of double diffusive convection. AEA, 540:A20, Apr. 2012.

J. Leconte and G. Chabrier. Layered convection as the origin of Saturn's luminosity anomaly. Nature Geoscience, 6(5):347-350, May 2013.

S. K. Leggett, M. C. Liu, T. J. Dupuy, C. V. Morley, M. S. Marley, and D. Saumon. Resolved Spectroscopy of the T8.5 and Y0-0.5 Binary WISEPC J121756.91+162640.2AB. ApJ, 780(1):62, Jan. 2014.

S. K. Leggett, C. V. Morley, M. S. Marley, and D. Saumon. Near-infrared Photometry of Y Dwarfs: Low Ammonia Abundance and the Onset of Water Clouds. The Astrophysical Journal, 799(1):37, Jan. 2015.

K. Lodders. Solar System Abundances and Condensation Temperatures of the Elements. ApJ, 591(2):1220-1247, July 2003.

P. W. Lucas, C. G. Tinney, B. Burningham, S. K. Leggett, D. J. Pinfield, R. Smart, H. R. A. Jones, F. Marocco, R. J. Barber, S. N. Yurchenko, J. Tennyson, M. Ishii, M. Tamura, A. C. Day-Jones, A. Adamson, F. Allard, and D. Homeier. The discovery of a very cool, very nearby brown dwarf in the Galactic plane. MNRAS, 408(1):L56-L60, Oct. 2010.

M. S. Marley, D. Saumon, and C. Goldblatt. A Patchy Cloud Model for the L to T Dwarf Transition. ApJL, 723(1):L117-L121, Nov. 2010.

C. V. Morley, J. J. Fortney, M. S. Marley, C. Visscher, D. Saumon, and S. K. Leggett. Neglected Clouds in T and Y Dwarf Atmospheres. ApJ, 756(2): 172, Sept. 2012.

C. V. Morley, M. S. Marley, J. J. Fortney, R. Lupu, D. Saumon, T. Greene, and K. Lodders. Water Clouds in Y Dwarfs and Exoplanets. ApJ, 787(1): 78, May 2014.

A. Padmanabhan, T. Tzanetakis, A. Chanda, and M. J. Thomson. Study of pressure broadening effects of $\mathrm{H} 2$ on $\mathrm{CO} 2$ and $\mathrm{CO}$ in the near infrared region between 6317 and $6335 \mathrm{~cm}-1$ at room temperature. Journal of Quantitative Spectroscopy and Radiative Transfer, 133:81-90, Jan. 2014.
J. Radigan. An Independent Analysis of the Brown Dwarf Atmosphere Monitoring (BAM) Data: Large-amplitude Variability is Rare Outside the L/T Transition. The Astrophysical Journal, 797(2):120, Dec. 2014.

S. Rahmstorf. Thermohaline circulation: The current climate. Nature, 421 (6):699, Feb. 2003.

R. A. Robie and D. R. Waldbaum. Thermodynamic properties of minerals and related substances at 298. 15/sup 0/K (25. 0/sup 0/C) and one atmosphere (1. 013 bars) pressure and at higher temperatures. Jan. 1968.

E. Rosenblum, P. Garaud, A. Traxler, and S. Stellmach. Turbulent Mixing and Layer Formation in Double-diffusive Convection: Three-dimensional Numerical Simulations and Theory. ApJ, 731(1):66, Apr. 2011.

D. Saumon, G. Chabrier, and H. M. van Horn. An Equation of State for Low-Mass Stars and Giant Planets. Astrophysical Journal Supplement v.99, 99:713, Aug. 1995.

D. Saumon, M. S. Marley, M. C. Cushing, S. K. Leggett, T. L. Roellig, K. Lodders, and R. S. Freedman. Ammonia as a Tracer of Chemical Equilibrium in the T7.5 Dwarf Gliese 570D. ApJ, 647(1):552-557, Aug. 2006.

D. Saumon, M. S. Marley, M. Abel, L. Frommhold, and R. S. Freedman. New H2 Collision-induced Absorption and NH3 Opacity and the Spectra of the Coolest Brown Dwarfs. ApJ, 750(1):74, May 2012.

C. M. Sharp and A. Burrows. Atomic and Molecular Opacities for Brown Dwarf and Giant Planet Atmospheres. The Astrophysical Journal Supplement Series, 168(1):140-166, Jan. 2007.

S. A. Tashkun and V. I. Perevalov. CDSD-4000: High-resolution, high-temperature carbon dioxide spectroscopic databank. Journal of Quantitative Spectroscopy and Radiative Transfer, 112:1403-1410, June 2011.

F. Thibault, J. Boissoles, R. Le Doucen, J. P. Bouanich, P. Arcas, and C. Boulet. Pressure induced shifts of CO2 lines - Measurements in the 00(0)3-00(0)0 band and theoretical analysis. Journal of Chemical Physics (ISSN 0021-9606), 96:4945-4953, Apr. 1992.

F. Thibault, B. Calil, J. Boissoles, and J. M. Launay. Experimental and theoretical $\mathrm{CO} 2 \mathrm{He}$ pressure broadening cross sections. Physical Chemistry Chemical Physics, 2:5404-5410, 2000.

C. G. Tinney, J. K. Faherty, J. D. Kirkpatrick, M. Cushing, C. V. Morley, and E. L. Wright. The Luminosities of the Coldest Brown Dwarfs. ApJ, 796 (1):39, Nov. 2014.

T. Tsuji, K. Ohnaka, and W. Aoki. Dust formation in stellar photospheres: a case of very low mass stars and a possible resolution on the effective temperature scale of M dwarfs. AEFA, 305:L1, Jan. 1996.

F. van Leeuwen. Validation of the new Hipparcos reduction. $A \mathcal{E} A, 474(2)$ : 653-664, Nov. 2007.

O. Venot, E. Hébrard, M. Agúndez, M. Dobrijevic, F. Selsis, F. Hersant, N. Iro, and R. Bounaceur. A chemical model for the atmosphere of hot Jupiters. AEA A, 546:43, Oct. 2012.

C. Wenger and J. P. Champion. Spherical Top Data System (STDS) software for the simulation of spherical top spectra. Journal of Quantitative Spectroscopy and Radiative Transfer, 59:471-480, May 1998.

Y. L. Yung, W. A. Drew, J. P. Pinto, and R. R. Friedl. Estimation of the reaction rate for the formation of $\mathrm{CH} 3 \mathrm{O}$ from $\mathrm{H}+\mathrm{H} 2 \mathrm{CO}$ - Implications for chemistry in the solar system. Icarus (ISSN 0019-1035), 73:516-526, Mar. 1988.

S. N. Yurchenko and J. Tennyson. ExoMol line lists - IV. The rotation-vibration spectrum of methane up to $1500 \mathrm{~K}$. MNRAS, 440(2): 1649-1661, May 2014. 